\title{
Continuous Time Random Walks in periodic systems: fluid limit and fractional differential equations on the circle
}

\author{
I Calvo ${ }^{1} \pm$, B A Carreras ${ }^{2}, \mathrm{R}$ Sánchez $^{3}$ and B Ph van Milligen ${ }^{1}$ \\ ${ }^{1}$ Laboratorio Nacional de Fusión, Asociación EURATOM-CIEMAT, E-28040 \\ Madrid, Spain \\ ${ }^{2}$ BACV Solutions Inc., Oak Ridge, TN 37830, U.S.A. \\ ${ }^{3}$ Fusion Energy Division, Oak Ridge National Laboratory, Oak Ridge, TN 37831, \\ U.S.A.
}

\begin{abstract}
In this article, the continuous time random walk on the circle is studied. We derive the corresponding generalized master equation and discuss the effects of topology, especially important when Lévy flights are allowed. Then, we work out the fluid limit equation, formulated in terms of the periodic version of the fractional Riemann-Liouville operators, for which we provide explicit expressions. Finally, we compute the propagator in some simple cases. The analysis presented herein should be relevant when investigating anomalous transport phenomena in systems with periodic dimensions.

PACS numbers: 05.40.Fb, 02.50.Ey, 05.60.Cd, 05.10.Gg
\end{abstract}

\section{Introduction}

Continuous Time Random Walks (CTRWs) ([1, [2]) are models describing the motion of individual particles (or any other conserved quantity) probabilistically. Since their introduction they have found many applications in physics, among other reasons, due to the fact that they allow exploring possibilities which go beyond the classical paradigm of diffusive transport. Their simplest realization, known as a separable CTRW, is defined in terms of two probability distribution functions (pdfs): the step-size pdf, $p$, and the waiting-time pdf, $\psi$. It is well-known that when the step-size pdf is Gaussian and the waiting-time pdf is exponential, the fluid limit (in which only long-time, large-distance information is retained) yields diffusive equations. These choices intrinsically imply the existence of finite characteristic length and time scales associated to the underlying microscopic transport mechanism.

However, there is a large number of physical, biological and social systems [3, 4, 5, 6] in which the dominant transport mechanism lacks either a well-defined characteristic length scale or a well-defined characteristic timescale or both. Instead, the characteristic

$\ddagger$ Corresponding author. E-mail: ivan.calvo@ciemat.es 
transport scales diverge with the system size and/or lifespan. For instance, this happens whenever transport takes place via avalanches. Transport events as a result have a maximum size that is only limited by the system size $L$, and a characteristic size that diverges with some power of $L$. This situation has been encountered, to list just a few examples, while investigating the transport of both particles and energy out of magnetically-confined fusion plasmas [7, 8, 9], in the propagation of forest fires [10], earthquakes [11], solar flares [12] and the transport of magnetic vortices in type-II superconductors [13]. It is possible to construct CTRW models that lack characteristic scales by choosing $p$ and $\psi$ from the family of stable Lévy distributions [14]. The fluid limit is then expressed in terms of transport equations that contain fractional differential operators [15], which are essentially non-local and non-Markovian. There is evidence, both numerical and experimental, that models generated in this manner provide a reasonably effective description of transport for some of the aforementioned problems [5, 6, 16, 17].

Although the formulation of CTRW models in finite systems is straightforward [18, 19], their fluid limit equations and associated propagators are much harder to obtain than in infinite domains. In this work we will study the formulation of CTRWs on a finite one-dimensional system with periodic boundary conditions (the unit circle) and work out its fluid limit in a rigorous way for an arbitrary stable Lévy distribution.

We envision that the formalism introduced in this paper might find application in systems in which particles or energy are amenable to trapping and/or avalanching processes along a periodic direction. A possible example might be the investigation of heat transport in single-wall carbon nanotube (SWNT) nanorings, which have gained recent attention due to their interesting transport properties [20], such as AharonovBohm effects, magnetotransport or establishment of persistent currents. Some molecular dynamical simulations have found that linear SWNTs seem to exhibit heat conductivities divergent with the nanotube length, which suggests that a characteristic length scale might be lacking [21. If these observations apply to nanorings as well, the periodic CTRW here presented might provide an adequate model for their effective description. Other possible applications might be to the modeling of some types of transport in turbulent plasmas confined in a (multi-)periodic system, such as those found in the (quasi-spherical) sun or in Earth-based (toroidal) magnetic confinement devices with interest for fusion energy production [22].

The paper is organized as follows. In Section 2 we derive the generalized master equation (GME) for a time-translational invariant, separable CTRW on the circle. In Section 3 we work out the fluid limit of such family of CTRWs introducing, in particular, the appropriate form of the Riemann-Liouville operators on the circle. The computation of the propagator of the fluid limit equations in the homogeneous case is presented in Section 4. We discuss its asymptotic behaviour and give analytical solutions for $\alpha=1,2$ in the Markovian case. Section 5 is devoted to the conclusions. Finally, Appendix A and Appendix B give a brief survey on stable Lévy distributions and fractional calculus, respectively. 


\section{CTRW on the circle. Generalized Master Equation}

We consider that the dynamics takes place on the unit circle, $S^{1}$, parameterized by $\theta \in[0,2 \pi)$. Sometimes it is useful to view the unit circle as $S^{1} \cong \mathbb{R} / 2 \pi$, where the quotient is taken with respect to the equivalence relation $x \sim x+2 \pi$ for any $x \in \mathbb{R}$. Functions on $S^{1}$ can then be identified with the set of periodic functions on $\mathbb{R}$ with period $2 \pi$. Explicitly, given $f: S^{1} \rightarrow \mathbb{R}$, its extension to a periodic function on $\mathbb{R}$ is

$$
\begin{aligned}
\breve{f}: & \mathbb{R} \\
x & \mapsto f([x]),
\end{aligned}
$$

where $[x]$ is the (unique) representative of the equivalence class of $x$ which belongs to $[0,2 \pi)$. Obviously, if $x \in[0,2 \pi), \breve{f}(x)=f(x)$. Finally, every function $f \in S^{1}$ considered in this paper is assumed to have a Fourier series expansion,

$$
f(\theta)=\frac{1}{2 \pi} \sum_{m=-\infty}^{\infty} f_{m} e^{-i m \theta},
$$

with coefficients given by the formula:

$$
f_{m}=\int_{0}^{2 \pi} f(\theta) e^{i m \theta} \mathrm{d} \theta .
$$

As mentioned above, we restrict ourselves to the case of separable CTRWs with time-translational invariance. This means that the CTRW is defined by two pdfs, $p(\Delta, \theta)$ and $\psi(\tau, \theta)$, called the step-size pdf and the waiting-time pdf, respectively. Here, $\Delta \in \mathbb{R}$, $\tau \geq 0$ and $\theta \in[0,2 \pi)$. $p$ gives the probability that a particle located at $\theta$ jumps to $\theta+\Delta$ after having waited at $\theta$ for a lapse of time $\tau$. Since $\Delta$ is unbounded, this may imply an arbitrary number of turns around the circle. Finally, $p$ and $\psi$ verify the normalization conditions,

$$
\begin{aligned}
& \int_{-\infty}^{\infty} p(\Delta, \theta) \mathrm{d} \Delta=1, \forall \theta \in[0,2 \pi) \\
& \int_{0}^{\infty} \psi(\tau, \theta) \mathrm{d} \tau=1, \quad \forall \theta \in[0,2 \pi) .
\end{aligned}
$$

We denote by $n(\theta, t)$ the density of particles normalized to the total number of

particles, i.e. $\int_{0}^{2 \pi} n(\theta, t) \mathrm{d} \theta=1, \forall t$. The GME for the time evolution of $n(\theta, t)$ corresponding to this class of CTRWs can be derived as follows [23, 18]. First, note that the normalized density of particles is equal to the probability of finding one particle at $\theta$ at time $t$. This probability can be rewritten as:

$$
n(\theta, t)=\int_{0}^{t} Q\left(\theta, t^{\prime}\right) \eta\left(\theta, t-t^{\prime}\right) \mathrm{d} t^{\prime}
$$

where $Q\left(\theta, t^{\prime}\right)$ gives the probability that the walker arrives at $\theta$ at time $t^{\prime}<t$, and $\eta(\theta, \tau)$ gives the probability of staying at $\theta$ for a lapse of time $\tau$. Clearly, one has:

$$
\eta(\theta, t)=1-\int_{0}^{t} \psi(\theta, \tau) \mathrm{d} \tau .
$$


Then, we rewrite:

$$
Q(\theta, t)=\sum_{j=0}^{\infty} Q^{j}(\theta, t)
$$

where $Q^{j}(\theta, t)$ denotes the probability that a single walker arrives at $\theta$ at time $t$ by performing exactly $j$ jumps. Obviously, for an arbitrary CTRW the function $Q^{j}(\theta, t)$ satisfies the following recurrence relation:

$$
Q^{j}(\theta, t)=\sum_{m=-\infty}^{\infty} \int_{0}^{2 \pi} \mathrm{d} \theta^{\prime} \int_{0}^{t} \mathrm{~d} t^{\prime} p\left(\theta-\theta^{\prime}+2 \pi m, \theta^{\prime}\right) \psi\left(t-t^{\prime}, \theta^{\prime}\right) Q^{j-1}\left(\theta^{\prime}, t^{\prime}\right),
$$

where the sum in front of the integral explicitly accounts for the fact that particles can arrive at $\theta$ from $\theta^{\prime}$ through jumps of length $\left|\theta-\theta^{\prime}+2 \pi m\right|, m \in \mathbb{Z} \cdot Q^{0}(\theta, t)=\delta(\theta) \delta(t)$ provides the needed initial condition. Adding over all possible values of $j$, we find an equation for $Q(\theta, t)$ :

$$
Q(\theta, t)-\delta(\theta) \delta(t)=\sum_{m=-\infty}^{\infty} \int_{0}^{2 \pi} \mathrm{d} \theta^{\prime} \int_{0}^{t} \mathrm{~d} t^{\prime} p\left(\theta-\theta^{\prime}+2 \pi m, \theta^{\prime}\right) \psi\left(t-t^{\prime}, \theta^{\prime}\right) Q\left(\theta^{\prime}, t^{\prime}\right)
$$

We proceed now to carry out the previously described periodic extension to the real line. Trivially, $\breve{p}, \breve{\psi}$ and $\breve{Q}$ also satisfy Eq. (10). In $\mathbb{R}$, we can make the change of variables $\tilde{\theta}=\theta^{\prime}-2 \pi m$ and use the periodicity properties of $\breve{p}, \breve{\psi}$ and $\breve{Q}$, together with the additivity of the integral with respect to the interval of integration, and convert Eq. (10) into:

$$
\breve{Q}(\theta, t)-\delta(\theta) \delta(t)=\int_{-\infty}^{\infty} \mathrm{d} \theta^{\prime} \int_{0}^{t} \mathrm{~d} t^{\prime} \breve{p}\left(\theta-\theta^{\prime}, \theta^{\prime}\right) \breve{\psi}\left(t-t^{\prime}, \theta^{\prime}\right) \breve{Q}\left(\theta^{\prime}, t^{\prime}\right) .
$$

This equation is formally identical to Eq. (12) from Ref. [18, which appears in the derivation of the GME in an infinite system. The main difference is that $\breve{Q}(\theta, t)$, whilst positive, is no longer a probability density in $\mathbb{R}$, since as a periodic function it is no longer integrable. This does not invalidate the procedure, because the convolution theorem still applies. Consequently, we can take the final result: Eq. (24) of Ref. [18]. Namely,

$\partial_{t} \breve{n}(\theta, t)=\int_{0}^{t} \mathrm{~d} t^{\prime} \int_{-\infty}^{\infty} \mathrm{d} \theta^{\prime} \breve{p}\left(\theta-\theta^{\prime}, \theta^{\prime}\right) \phi\left(\theta^{\prime}, t-t^{\prime}\right) \breve{n}\left(\theta^{\prime}, t^{\prime}\right)-\int_{0}^{t} \phi\left(\theta, t-t^{\prime}\right) \breve{n}\left(\theta, t^{\prime}\right) \mathrm{d} t^{\prime}$,

where $\phi$ is also a periodic function defined by:

$$
\phi(\theta, t)=\mathcal{L}^{-1}\left[\frac{\mathcal{L}[\breve{\psi}(\theta, t)]}{\mathcal{L}[\breve{\eta}(\theta, t)]}\right]
$$

and $\mathcal{L}[\cdot]$ stands for the Laplace transform with respect to $t$.

Again, $\breve{n}(\theta, t)$ is not a probability density function in $\mathbb{R}$ even when positive because it is periodic and thus non-integrable. We need to restrict the integrals to $[0,2 \pi)$ to recover the probabilistic interpretation. To do so, we simply reverse the manipulations which led us from (10) to (11), recasting the GME (12) as

$\partial_{t} n(\theta, t)=\int_{0}^{t} \mathrm{~d} t^{\prime} \int_{0}^{2 \pi} \mathrm{d} \theta^{\prime} \bar{p}\left(\theta-\theta^{\prime}, \theta^{\prime}\right) \phi\left(\theta^{\prime}, t-t^{\prime}\right) n\left(\theta^{\prime}, t^{\prime}\right)-\int_{0}^{t} \phi\left(\theta, t-t^{\prime}\right) n\left(\theta, t^{\prime}\right) \mathrm{d} t^{\prime}$, 
with

$$
\bar{p}\left(\theta, \theta^{\prime}\right)=\sum_{m=-\infty}^{\infty} p\left(\theta+2 \pi m, \theta^{\prime}\right) .
$$

From this expression it is obvious that $\bar{p}$ is positive-definite and periodic in its first argument, $\bar{p}\left(0, \theta^{\prime}\right)=\bar{p}\left(2 \pi, \theta^{\prime}\right)$, for any $\theta^{\prime}$. Furthermore, the normalization of $p$ in $\mathbb{R}$ implies the normalization of $\bar{p}$ in $S^{1}$,

$$
\int_{0}^{2 \pi} \bar{p}\left(\theta, \theta^{\prime}\right) \mathrm{d} \theta=1, \forall \theta^{\prime} \in[0,2 \pi),
$$

so that $\bar{p}$ is a probability distribution on the circle. Hence, the GME (14) is written in terms of functions on $S^{1}$ and makes manifest that the dynamics of $n(\theta, t)$ is well-defined on the circle. It is worth pointing out that (15) expresses the fact that particles can wind around the circle an arbitrary number of times, and hence is a consequence of the non-trivial topology of the manifold on which the CTRW takes place. Actually, $\bar{p}$ shows up as the effective step-size pdf on the circle and is related to the original step-size pdf, $p$, through (15). This relation is in fact the ballooning transform that was introduced in the plasma physics literature in the late 70s to deal with PDEs formulated on toroidal geometries [24]. Figs. 1(a) and 1(b) compare $p$ and $\bar{p}$ when $p$ is a Gaussian and a Cauchy distribution (see Appendix A), respectively. As expected, the effect of topology is much more relevant for Lévy distributions with algebraic tails than for the Gaussian.

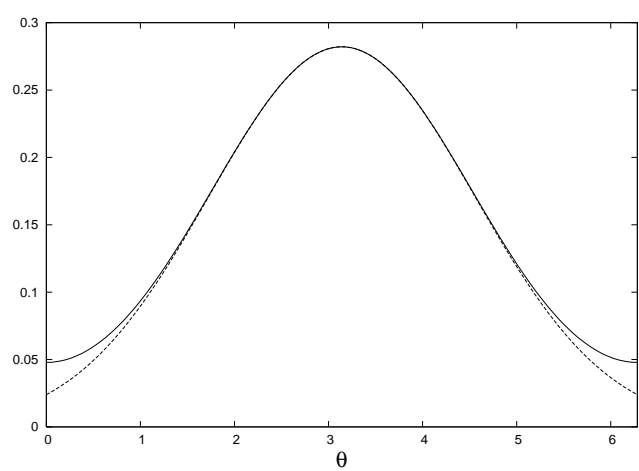

(a) Gaussian

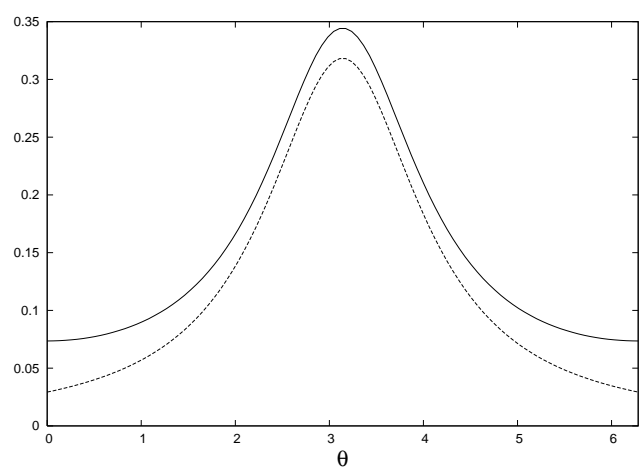

(b) Cauchy

Figure 1. Comparison of $p$ (dashed) and its associated $\bar{p}$ (solid). (a) $p$ is a symmetric Gaussian distribution (i.e. $\alpha=2$ ). (b) $p$ is a symmetric Cauchy distribution (i.e. $\alpha=1$ ). In both subfigures, $p$ has $\sigma=1$ and is centered at $\theta=\pi$.

\section{Fluid limit of the GME. Fractional differential operators on the circle}

Since we will be especially interested in the fluid limit of the CTRW when $p$ and/or $\psi$ are Lévy distributions (which only have a finite number of finite integer moments), we cannot carry out the calculation by the standard method of expanding the GME using moments of these distributions. The appropriate procedure involves going to the Fourier 
(for space) and Laplace (for time) domains. The fluid limit, which implies retaining only the dynamical information pertaining to the longer times and larger distances, corresponds to taking the limits $k \rightarrow 0, s \rightarrow 0$, where $k$ and $s$ stand respectively for the Fourier and Laplace variables.

Since $\bar{p}$ is periodic with period $2 \pi$, we can expand it in a Fourier series with respect to its first argument:

$$
\bar{p}\left(\theta, \theta^{\prime}\right)=\frac{1}{2 \pi} \sum_{m=-\infty}^{\infty} \bar{p}_{m}\left(\theta^{\prime}\right) e^{-i m \theta} .
$$

Recalling the definition of $\bar{p}$ in terms of $p$, the coefficients of this expansion are:

$$
\begin{aligned}
\bar{p}_{m}\left(\theta^{\prime}\right) & =\int_{0}^{2 \pi} \bar{p}\left(\theta, \theta^{\prime}\right) e^{i m \theta} \mathrm{d} \theta=\sum_{m^{\prime}=-\infty}^{\infty} \int_{0}^{2 \pi} p\left(\theta+2 \pi m^{\prime}, \theta^{\prime}\right) e^{i m \theta} \mathrm{d} \theta \\
& =\sum_{m^{\prime}=-\infty}^{\infty} \int_{2 \pi m^{\prime}}^{2 \pi\left(m^{\prime}+1\right)} p\left(\tilde{\theta}, \theta^{\prime}\right) e^{i m \tilde{\theta}} e^{-i 2 \pi m m^{\prime}} \mathrm{d} \tilde{\theta}=\sum_{m^{\prime}=-\infty}^{\infty} \int_{2 \pi m^{\prime}}^{2 \pi\left(m^{\prime}+1\right)} p\left(\tilde{\theta}, \theta^{\prime}\right) e^{i m \tilde{\theta}} \mathrm{d} \tilde{\theta} \\
& =\int_{-\infty}^{\infty} p\left(\tilde{\theta}, \theta^{\prime}\right) e^{i m \tilde{\theta}} \mathrm{d} \tilde{\theta}=\hat{p}\left(m ; \theta^{\prime}\right) .
\end{aligned}
$$

where in the third equality we have performed the change of variables $\tilde{\theta}=\theta+2 \pi m^{\prime}$ and in the fourth equality we have used that $e^{-i 2 \pi m m^{\prime}}=1$. Hence, the $m$-th coefficient in the Fourier expansion of $\bar{p}$ with respect to its first argument is exactly the value of the Fourier transform of $p$ with respect to its first argument at $k=m$. This relation is characteristic of the aforementioned ballooning transform [24].

Following Ref. [25], we proceed by inserting this expansion in (14),

$$
\begin{aligned}
\partial_{t} n(\theta, t) & =\frac{1}{2 \pi} \sum_{m^{\prime}=-\infty}^{\infty} \int_{0}^{t} \mathrm{~d} t^{\prime} \int_{0}^{2 \pi} \mathrm{d} \theta^{\prime} \phi\left(\theta^{\prime}, t-t^{\prime}\right) \bar{p}_{m^{\prime}}\left(\theta^{\prime}\right) e^{-i m^{\prime}\left(\theta-\theta^{\prime}\right)} n\left(\theta^{\prime}, t^{\prime}\right) \\
& -\int_{0}^{t} \phi\left(\theta, t-t^{\prime}\right) n\left(\theta, t^{\prime}\right) \mathrm{d} t^{\prime}
\end{aligned}
$$

Now, we expand both sides in Fourier series with respect to $\theta$ and equate coefficients,

$$
\begin{aligned}
\partial_{t} n_{m}(t) & =\frac{1}{2 \pi} \sum_{m^{\prime}=-\infty}^{\infty} \int_{0}^{2 \pi} \mathrm{d} \theta e^{i\left(m-m^{\prime}\right) \theta} \int_{0}^{t} \mathrm{~d} t^{\prime} \int_{0}^{2 \pi} \mathrm{d} \theta^{\prime} \phi\left(\theta^{\prime}, t-t^{\prime}\right) \bar{p}_{m^{\prime}}\left(\theta^{\prime}\right) e^{i m^{\prime} \theta^{\prime}} n\left(\theta^{\prime}, t^{\prime}\right) \\
& -\int_{0}^{2 \pi} \mathrm{d} \theta e^{i m \theta} \int_{0}^{t} \phi\left(\theta, t-t^{\prime}\right) n\left(\theta, t^{\prime}\right) \mathrm{d} t^{\prime} .
\end{aligned}
$$

Since $\int_{0}^{2 \pi} e^{i\left(m-m^{\prime}\right) \theta} \mathrm{d} \theta=2 \pi \delta_{m m^{\prime}}$ and $\bar{p}_{m}\left(\theta^{\prime}\right)=\hat{p}\left(m ; \theta^{\prime}\right)$ we obtain:

$$
\partial_{t} n_{m}(t)=\int_{0}^{t} \mathrm{~d} t^{\prime} \int_{0}^{2 \pi} \mathrm{d} \theta^{\prime} \phi\left(\theta^{\prime}, t-t^{\prime}\right)\left(\hat{p}\left(m ; \theta^{\prime}\right)-1\right) e^{i m \theta^{\prime}} n\left(\theta^{\prime}, t^{\prime}\right) .
$$

Let $\Lambda(k ; \theta)$ be the characteristic exponent of $p(\Delta, \theta)$, i.e. $\hat{p}(k ; \theta)=\exp \Lambda(k ; \theta)$. The fluid limit is defined by taking $\hat{p}(k ; \theta) \approx 1+\Lambda(k ; \theta)$. Therefore, Eq. (21) in the fluid limit is written as

$$
\partial_{t} n_{m}(t)=\int_{0}^{2 \pi} \mathrm{d} \theta^{\prime} \Lambda\left(m ; \theta^{\prime}\right) e^{i m \theta^{\prime}} \int_{0}^{t} \mathrm{~d} t^{\prime} \phi\left(\theta^{\prime}, t-t^{\prime}\right) n\left(\theta^{\prime}, t^{\prime}\right) .
$$


At this point we need to make choices for the step-size and waiting-time pdfs. A natural choice is to take stable Lévy distributions, which satisfy the central limit theorem [14]. Thus, following Ref. [26], we will assume first that the waiting-time pdf is a positive extremal Lévy distribution (see Appendix A). Performing a (non-analytic, in general) expansion of the Laplace transform of $\psi$ around $s=0$ and keeping the lowest order terms:

$$
\tilde{\psi}(s) \approx 1-A_{\gamma}^{-1} \tau^{\gamma} s^{\gamma}, \quad \gamma \in(0,1]
$$

where $A_{\gamma}=\cos (\pi \gamma / 2)$ if $\gamma \in(0,1)$ and $A_{\gamma}=1$ if $\gamma=1$, which corresponds to an exponential waiting-time pdf. The convolution in the second integral of (22) in the fluid limit yields:

$$
\mathcal{L}\left[\int_{0}^{t} \mathrm{~d} t^{\prime} \phi\left(\theta^{\prime}, t-t^{\prime}\right) n\left(\theta^{\prime}, t^{\prime}\right)\right] \approx A_{\gamma} \tau^{-\gamma} s^{1-\gamma} \tilde{n}\left(\theta^{\prime}, s\right)
$$

where we have made use of (A.3) . Then, Eq. (22) in Laplace space reads:

$$
s \tilde{n}_{m}(s)-n_{m}(0)=A_{\gamma} \tau^{-\gamma} s^{1-\gamma} \int_{0}^{2 \pi} \Lambda\left(m ; \theta^{\prime}\right) \tilde{n}\left(\theta^{\prime} ; s\right) e^{i m \theta^{\prime}} \mathrm{d} \theta^{\prime} .
$$

Now, multiplying both sides by $s^{\gamma-1}$, using (B.2) and (B.3) and Laplace inverting:

$$
\frac{\mathrm{d}^{\gamma}}{\mathrm{d} t^{\gamma}} n_{m}(t)=A_{\gamma} \tau^{-\gamma} \int_{0}^{2 \pi} \Lambda\left(m ; \theta^{\prime}\right) n\left(\theta^{\prime}, t\right) e^{i m \theta^{\prime}} \mathrm{d} \theta^{\prime}
$$

As for the spatial part, we will consider the case in which $\Lambda(k ; \theta)$ is the characteristic exponent of a general Lévy distribution (see Appendix A) with parameters dependent on $\theta$ :

$\Lambda(k ; \theta)= \begin{cases}-\sigma^{\alpha}(\theta)|k|^{\alpha}\left[1-i \beta(\theta) \operatorname{sign}(k) \tan \left(\frac{\pi \alpha}{2}\right)\right]+i \mu(\theta) k & \alpha \in(0,1) \cup(1,2], \\ -\sigma(\theta)|k|+i \mu(\theta) k & \alpha=1,\end{cases}$

restricting ourselves to $\beta \equiv 0$ for $\alpha=1$.

Let us work out the case $\alpha \neq 1$. Eq. (26) becomes

$$
\begin{aligned}
\frac{\mathrm{d}^{\gamma}}{\mathrm{d} t^{\gamma}} n_{m}(t) & =-A_{\gamma} \tau^{-\gamma}\left(\int_{0}^{2 \pi} \sigma^{\alpha}\left(\theta^{\prime}\right)|m|^{\alpha}\left[1-i \beta\left(\theta^{\prime}\right) \operatorname{sign}(m) \tan \left(\frac{\pi \alpha}{2}\right)\right] e^{i m \theta^{\prime}} n\left(\theta^{\prime}, t\right) \mathrm{d} \theta^{\prime}\right. \\
& \left.+\int_{0}^{2 \pi} i m \mu\left(\theta^{\prime}\right) e^{i m \theta^{\prime}} n\left(\theta^{\prime}, t\right) \mathrm{d} \theta^{\prime}\right) .
\end{aligned}
$$

or equivalently,

$\frac{\mathrm{d}^{\gamma}}{\mathrm{d} t^{\gamma}} n_{m}(t)=-\frac{A_{\gamma}}{\tau^{\gamma}}\left[|m|^{\alpha}\left(\sigma^{\alpha} n\right)_{m}-i \tan \left(\frac{\pi \alpha}{2}\right)|m|^{\alpha} \operatorname{sign}(m)\left(\beta \sigma^{\alpha} n\right)_{m}+i m(\mu n)_{m}\right](t)$.

The inverse Fourier transform of Eq. (29) is the desired fluid limit of the CTRW on the circle. The analogue of (29) in an infinite system is Fourier inverted by means of the Riemann-Liouville operators defined in Appendix B. The following theorem (see [27] for related results) yields the suitable generalization of the Riemann-Liouville operators on the circle. 
Fluid limit of Continuous Time Random Walks on the circle

Theorem: Let $f$ be a sufficiently well-behaved function on $S^{1}, \alpha \in(0,1) \cup(1,2)$, and define the operators

$$
\begin{aligned}
& { }_{0} \mathcal{D}^{\alpha} f(\theta)={ }_{0} D^{\alpha} f(\theta)+\frac{1}{(2 \pi)^{\alpha+1} \Gamma(-\alpha)} \int_{0}^{2 \pi} f\left(\theta^{\prime}\right) \zeta\left(1+\alpha, 1+\frac{\theta-\theta^{\prime}}{2 \pi}\right) \mathrm{d} \theta^{\prime} \\
& { }^{2 \pi} \mathcal{D}^{\alpha} f(\theta)={ }^{2 \pi} D^{\alpha} f(\theta)-\frac{1}{(2 \pi)^{\alpha+1} \Gamma(-\alpha)} \int_{0}^{2 \pi} f\left(\theta^{\prime}\right) \zeta\left(1+\alpha, 1+\frac{\theta^{\prime}-\theta}{2 \pi}\right) \mathrm{d} \theta^{\prime},
\end{aligned}
$$

where ${ }_{0} D^{\alpha}$ and ${ }^{2 \pi} D^{\alpha}$ are the Riemann-Liouville operators defined in (B.1), $q$ is the smallest integer greater than $\alpha$ and $\zeta$ is the Hurwitz zeta function,

$$
\zeta(s, a)=\sum_{m=0}^{\infty} \frac{1}{(m+a)^{s}}, \quad \operatorname{Re}(s)>1, m+a \neq 0 .
$$

Then,

$$
\left({ }_{0} \mathcal{D}^{\alpha} f\right)_{m}=(-i m)^{\alpha} f_{m}, \quad\left({ }^{2 \pi} \mathcal{D}^{\alpha} f\right)_{m}=(i m)^{\alpha} f_{m}
$$

Proof:

First, notice that it is enough to show that ${ }_{0} \mathcal{D}^{\alpha} e^{i m \theta}=(i m)^{\alpha} e^{i m \theta}$ and ${ }^{2 \pi} \mathcal{D}^{\alpha} e^{i m \theta}=$ $(-i m)^{\alpha} e^{i m \theta}$. Let us give the proof for ${ }_{0} \mathcal{D}^{\alpha}$. By the definition of the Hurwitz zeta function and recalling that $\Gamma(z+1)=z \Gamma(z)$,

$$
\begin{aligned}
{ }_{0} \mathcal{D}^{\alpha} f(\theta) & ={ }_{0} D^{\alpha} f(\theta)+\frac{1}{\Gamma(-\alpha)} \sum_{m=0}^{\infty} \int_{0}^{2 \pi} \frac{f\left(\theta^{\prime}\right)}{\left(2 \pi(m+1)+\theta-\theta^{\prime}\right)^{1+\alpha}} \mathrm{d} \theta^{\prime} \\
& ={ }_{0} D^{\alpha} f(\theta)+\frac{1}{\Gamma(q-\alpha)} \sum_{m=0}^{\infty} \frac{\mathrm{d}^{q}}{\mathrm{~d} \theta^{q}} \int_{0}^{2 \pi} \frac{f\left(\theta^{\prime}\right)}{\left(2 \pi(m+1)+\theta-\theta^{\prime}\right)^{1+\alpha-q}} \mathrm{~d} \theta^{\prime} .
\end{aligned}
$$

Performing the change of variables $\tilde{\theta}=\theta^{\prime}-2 \pi(m+1)$, and using the periodicity of $\breve{f}$ we have:

$$
\begin{aligned}
& \sum_{m=0}^{\infty} \frac{\mathrm{d}^{q}}{\mathrm{~d} \theta^{q}} \int_{0}^{2 \pi} \frac{f\left(\theta^{\prime}\right)}{\left(2 \pi(m+1)+\theta-\theta^{\prime}\right)^{1+\alpha-q}} \mathrm{~d} \theta^{\prime}= \\
& \sum_{m=0}^{\infty} \frac{\mathrm{d}^{q}}{\mathrm{~d} \theta^{q}} \int_{-2 \pi(m+1)}^{-2 \pi m} \frac{\breve{f}(\tilde{\theta}+2 \pi(m+1))}{(\theta-\tilde{\theta})^{1+\alpha-q}} \mathrm{~d} \tilde{\theta}=\lim _{a \rightarrow-\infty} \frac{\mathrm{d}^{q}}{\mathrm{~d} \theta^{q}} \int_{a}^{0} \frac{\breve{f}(\tilde{\theta})}{(\theta-\tilde{\theta})^{1+\alpha-q}} \mathrm{~d} \tilde{\theta} .
\end{aligned}
$$

Recalling the definition (B.1) of the Riemann-Liouville operators we immediately obtain that

$$
{ }_{0} \mathcal{D}^{\alpha} f(\theta)={ }_{-\infty} D^{\alpha} \breve{f}(\theta) .
$$

Since ${ }_{-\infty} D^{\alpha} e^{i m \theta}=(i m)^{\alpha} e^{i m \theta}$, the result follows. An analogous calculation shows that ${ }^{2 \pi} \mathcal{D}^{\alpha} e^{i m \theta}=(-i m)^{\alpha} e^{i m \theta}$.

Let us go back to Eq. (29). Making use of the Theorem and the identities

$$
|m|^{\alpha}=\frac{(i m)^{\alpha}+(-i m)^{\alpha}}{2 \cos (\pi \alpha / 2)}, \quad|m|^{\alpha} \operatorname{sgn}(m)=\frac{(i m)^{\alpha}-(-i m)^{\alpha}}{2 i \sin (\pi \alpha / 2)}
$$


for $\alpha \in(0,1) \cup(1,2)$, we can Fourier-invert Eq. (29) in terms of the operators defined in (30):

$$
\begin{aligned}
\frac{\mathrm{d}^{\gamma}}{\mathrm{d} t^{\gamma}} n(\theta, t) & =-\frac{A_{\gamma}}{2 \tau^{\gamma} \cos (\pi \alpha / 2)}\left[{ }_{0} \mathcal{D}_{\theta}^{\alpha}\left((1+\beta) \sigma^{\alpha} n\right)+{ }^{2 \pi} \mathcal{D}_{\theta}^{\alpha}\left((1-\beta) \sigma^{\alpha} n\right)\right](\theta, t) \\
& +\frac{A_{\gamma}}{\tau^{\gamma}} \partial_{\theta}(\mu n)(\theta, t),
\end{aligned}
$$

which is the fluid equation corresponding to the CTRW on the circle. The cases $\alpha=1$ (with $\beta \equiv 0$ ) and $\alpha=2$ can be obtained, respectively, as the limits $\alpha \rightarrow 1^{+}$and $\alpha \rightarrow 2^{-}$ of the above expressions.

\section{Propagator of the fluid limit equations in the homogeneous case}

In this section we will particularize the above results to homogeneous systems and will compute the propagator (also known as the fundamental solution) of the fluid limit equations of the CTRW on the circle. That is, the solution with initial condition

$$
n(\theta, 0)=\sum_{m=-\infty}^{\infty} \delta(\theta-2 \pi m)
$$

sometimes called the Dirac comb. Its Fourier expansion is

$$
n(\theta, 0)=\frac{1}{2 \pi} \sum_{m=-\infty}^{\infty} e^{-i m \theta}
$$

i.e. $\left.n_{m}(t)\right|_{t=0}=1, \forall m \in \mathbb{Z}$.

If the system is homogeneous Eq. (26) takes the simple form

$$
\frac{\mathrm{d}^{\gamma}}{\mathrm{d} t^{\gamma}} n_{m}(t)=A_{\gamma} \tau^{-\gamma} \Lambda(m) n_{m}(t)
$$

which after Laplace transforming allows to give the solution of the propagator as

$$
n(\theta, t)=\frac{1}{2 \pi} \sum_{m=-\infty}^{\infty} \mathcal{L}^{-1}\left[\frac{s^{\gamma-1}}{s^{\gamma}-A_{\gamma} \tau^{-\gamma} \Lambda(m)}\right](t) e^{-i m \theta}
$$

We can make further analytical progress by using the so-called 1-parameter MittagLeffler functions, defined by [15]:

$$
E_{p}(z):=\sum_{j=0}^{\infty} \frac{z^{j}}{\Gamma(p j+1)},
$$

for $p>0$. These functions provide a suitable generalization of the exponential function (indeed, note that $E_{1}(z)=\exp (z)$ ) which is useful to us because of the property:

$$
\mathcal{L}^{-1}\left[\frac{s^{p-1}}{s^{p} \mp a}\right](t)=E_{p}\left( \pm a t^{\gamma}\right) .
$$

Thus, Eq. (41) can be recast as:

$$
n(\theta, t)=\frac{1}{2 \pi} \sum_{m=-\infty}^{\infty} E_{\gamma}\left(A_{\gamma} \Lambda(m)(t / \tau)^{\gamma}\right) e^{-i m \theta} .
$$


In the Markovian case, $\gamma=1$, we can produce more explicit expressions. By setting $\gamma=1$ in (44) we obtain:

$$
n(\theta, t)=\frac{1}{2 \pi} \sum_{m=-\infty}^{\infty} e^{\Lambda(m) t / \tau} e^{-i m \theta} .
$$

In particular, if $\Lambda(m)$ is the characteristic exponent of a stable Lévy distribution with $\beta=0$,

$$
n(\theta, t)=\frac{1}{2 \pi} \sum_{m=-\infty}^{\infty} e^{\left(-\sigma^{\alpha}|m|^{\alpha}+i \mu m\right) t / \tau} e^{-i m \theta}, \alpha \in(0,2] .
$$

In the limit $t \rightarrow \infty, n(\theta, t) \rightarrow 1 / 2 \pi$, as required by the condition of conservation of probability.

Given a symmetric Lévy distribution $(\beta=\mu=0)$, it is well-known that the fluid limit propagator in an infinite, homogeneous system is invariant under rescaling of the so-called self-similarity variable, $\chi:=x^{\alpha} / t^{\gamma}$, and the propagator (with initial condition localized at the origin) at $x=0$ decays as $t^{-1 / \alpha}$. Things work quite differently in a periodic system, in which the notion of self-similiarity does not make sense. For the time evolution of the propagator on the circle $(\beta=\mu=0)$ at $\theta=0, n(0, t)$, there exist two distinct regimes:

- Long-time asymptotics: If $t>>\tau / \sigma^{\alpha}$ it is clear that

$$
n(0, t) \sim \frac{1}{2 \pi}\left(1+2 e^{-\sigma^{\alpha} t / \tau}\right) .
$$

Thus $n(0, t)$ decays exponentially for any $\alpha$.

- Intermediate asymptotics: For $t<<\tau / \sigma^{\alpha}$, we can approximate the infinite sum which defines $n(0, t)$ by an integral,

$$
n(0, t)=\frac{1}{2 \pi} \sum_{m=-\infty}^{\infty} e^{-\sigma^{\alpha}|m|^{\alpha} t / \tau} \approx \frac{1}{2 \pi} \int_{-\infty}^{\infty} e^{-\sigma^{\alpha}|u|^{\alpha} t / \tau} \mathrm{d} u \propto t^{-1 / \alpha} .
$$

Hence, at short times the $\alpha$-dependent algebraic decay characteristic of the propagator in infinite systems is recovered.

In the next subsections we work out the special cases $\alpha=2$ and $\alpha=1$, for which we can provide analytical solutions for the propagator.

\subsection{Diffusive transport: $\alpha=2$}

For $\alpha=2$ the step-size pdf becomes a Gaussian distribution and (46) yields:

$$
n(\theta, t)=\frac{1}{2 \pi} \sum_{m=-\infty}^{\infty} \exp \left(-m^{2} \sigma^{2} t / \tau+i m(\mu t / \tau-\theta)\right),
$$

which can be written in terms of the third Jacobi theta function [28]:

$$
\vartheta_{3}(z, q)=1+2 \sum_{m=1}^{\infty} q^{m^{2}} \cos (2 m z), \quad|q|<1, z \in \mathbb{C} .
$$


Namely,

$$
n(\theta, t)=\frac{1}{2 \pi} \vartheta_{3}\left(\frac{1}{2}\left(\frac{\mu t}{\tau}-\theta\right), e^{-\sigma^{2} t / \tau}\right)
$$

which is the solution of the heat equation in a periodic system.

\subsection{Cauchy distribution: $\alpha=1$}

Let us consider the case $\alpha=1, \beta=0$, which corresponds to a Cauchy step-size pdf. In this situation Eq. (46) becomes:

$$
n(\theta, t)=\frac{1}{2 \pi} \sum_{m=-\infty}^{\infty} e^{-|m| \sigma t / \tau+i m(\mu t / \tau-\theta)} .
$$

We can split the sum as

$$
n(\theta, t)=\frac{1}{2 \pi}\left(\sum_{m=0}^{\infty} e^{(-\sigma t / \tau+i(\mu t / \tau-\theta)) m}+\sum_{m=1}^{\infty} e^{(-\sigma t / \tau-i(\mu t / \tau-\theta)) m}\right),
$$

so that both terms are geometric series with ratios $\exp (-\sigma t / \tau+i(\mu t / \tau-\theta))$ and $\exp (-\sigma t / \tau-i(\mu t / \tau-\theta))$, respectively. Then,

$$
\begin{aligned}
\sum_{m=0}^{\infty} e^{(-\sigma t / \tau+i(\mu t / \tau-\theta)) m} & =\frac{1}{1-\exp (-\sigma t / \tau+i(\mu t / \tau-\theta))}, \\
\sum_{m=1}^{\infty} e^{(-\sigma t / \tau-i(\mu t / \tau-\theta)) m} & =\frac{1}{1-\exp (-\sigma t / \tau-i(\mu t / \tau-\theta))}-1
\end{aligned}
$$

and we can give a closed expression for the propagator:

$$
n(\theta, t)=\frac{1}{2 \pi} \frac{\sinh (\sigma t / \tau)}{\cosh (\sigma t / \tau)-\cos (\mu t / \tau-\theta)} .
$$

It is instructive to work out the approximate form of the propagator for small $t$ and in the vicinity of $\theta=0$ directly from Eq. (55), obtaining:

$$
n(\theta, t) \approx \frac{1}{\pi} \frac{\sigma t / \tau}{(\sigma t / \tau)^{2}+(\mu t / \tau-\theta)^{2}} .
$$

This formally coincides with the propagator in an infinite system. In particular, $n(0, t) \propto 1 / t$ for small $t$, in agreement with the general result (48).

\section{Conclusions}

Although there are numerous results concerning CTRWs in infinite systems, much work still remains to be done on their finite-size formulation. In this paper we have performed a detailed study of time translationally invariant, spatially inhomogeneous, separable CTRWs on the circle, in order to investigate how the topology can affect the form of the Generalized Master Equation and the corresponding fluid limit equations.

We have found that, in order to formulate the CTRW on the circle, a non-trivial transformation of the step-size pdf must be carried out which is strongly reminiscent 
of the ballooning transform familiar to plasma physicists. During the derivation of the fluid limit of these periodic CTRWs, it was shown that the standard RiemannLiouville operators need to be reformulated on the circle. We have provided original expressions for them. In the homogeneous, Markovian case, we have been able to compute the propagators of the resulting fluid limit equations, even in closed form for some simple cases. Similar calculations may be performed in the non-Markovian case, in a straightforward manner, by using Mittag-Leffler functions. Finally, we have also hinted how these propagators may prove useful when determining the characteristic exponents in experimental situations, by focusing on their short-time behaviour. We feel that the formalism developed herein will find an application in physical systems in which both periodicity and anomalous transport phenomena are encountered. As mentioned in the Introduction, this could be the case of systems like SWNT nanorings and gravitationally or magnetically confined plasmas.

Acknowledgements: Research sponsored by DGICYT (Dirección General de Investigaciones Científicas y Tecnológicas) of Spain under Project No. ENE2004-04319. B. A. C. gratefully acknowledges the hospitality of CIEMAT during some of the phases of this work. Part of this research was sponsored by the Laboratory Research and Development Program of Oak Ridge National Laboratory, managed by UT-Battelle, LLC, for the US Department of Energy under contract number DE-AC05-00OR22725.

\section{Appendix A. Lévy skew alpha-stable distributions}

The family of Lévy skew alpha-stable distributions (or simply stable distributions, or Lévy distributions) is parameterized by four real numbers $\alpha \in(0,2], \beta \in[-1,1], \sigma>0$, and $\mu \in \mathbb{R}$. Their characteristic function (i.e. their Fourier transform) is given by [14]:

$\hat{S}(\alpha, \beta, \sigma, \mu)(k)= \begin{cases}\exp \left(-\sigma^{\alpha}|k|^{\alpha}\left[1-i \beta \operatorname{sign}(k) \tan \left(\frac{\pi \alpha}{2}\right)\right]+i \mu k\right) & \alpha \neq 1, \\ \exp \left(-\sigma|k|\left[1+i \beta \frac{2}{\pi} \operatorname{sign}(k) \ln |k|\right]+i \mu k\right) & \alpha=1 .\end{cases}$

According to the Generalized Central Limit Theorem ([29, 14]), stable distributions are the only possible distributions with a domain of attraction. The index of stability, $\alpha$, is related to the asymptotic behaviour of $S(\alpha, \beta, \sigma, \mu)(x)$ at large $x$ :

$S(\alpha, \beta, \sigma, \mu)(x)= \begin{cases}C_{\alpha}\left(\frac{1-\beta}{2}\right) \sigma^{\alpha}|x|^{-1-\alpha} & x \rightarrow-\infty, \\ C_{\alpha}\left(\frac{1+\beta}{2}\right) \sigma^{\alpha}|x|^{-1-\alpha} & x \rightarrow \infty,\end{cases}$

for $\alpha \in(0,2)$. For $\alpha=2, S(2, \beta, \sigma, \mu)$ is a Gaussian distribution.

The skewness parameter, $\beta$, measures the asymmetry of the distribution. If $\beta=0$, $S(\alpha, 0, \sigma, \mu)$ is symmetric with respect to $\mu$, whereas if $\beta= \pm 1$ the distribution is said to be extremal. Extremal distributions when $0<\alpha<1$ are especially interesting because they are one-sided: they are only defined for $x>0$ if $\beta=1$ and for $x<0$ if $\beta=-1$. Their Laplace transform is given by $(\mu=0)$ :

$$
\tilde{S}(\alpha, 1, \sigma, 0)(s)=\exp \left(-\frac{\sigma^{\alpha}}{\cos (\pi \alpha / 2)} s^{\alpha}\right) .
$$




\section{Appendix B. Fractional differential operators on the real line}

The Riemann-Liouville fractional differential operators are defined as [30], [15]:

$$
\begin{aligned}
{ }_{a} D_{x}^{\alpha} f: & :=\frac{1}{\Gamma(m-\alpha)} \frac{\mathrm{d}^{m}}{\mathrm{~d} x^{m}} \int_{a}^{x} \frac{f\left(x^{\prime}\right)}{\left(x-x^{\prime}\right)^{\alpha-m+1}} \mathrm{~d} x^{\prime}, \\
{ }^{b} D_{x}^{\alpha} f: & =\frac{(-1)^{m+1}}{\Gamma(m-\alpha)} \frac{\mathrm{d}^{m}}{\mathrm{~d} x^{m}} \int_{x}^{b} \frac{f\left(x^{\prime}\right)}{\left(x^{\prime}-x\right)^{\alpha-m+1}} \mathrm{~d} x^{\prime},
\end{aligned}
$$

where $\Gamma$ is the Euler Gamma function and $m \in \mathbb{Z}$ is the ceiling of $\alpha$ (i.e. the smallest integer greater than or equal to $\alpha$ ). When $a \rightarrow-\infty$ and $b \rightarrow \infty$ the action of the Riemman-Liouville operators in Fourier space satisfies the important property:

$$
\begin{aligned}
& \mathcal{F}\left[{ }_{-\infty} D_{x}^{\alpha} f\right](k)=(-i k)^{\alpha} \mathcal{F}[f](k) \\
& \mathcal{F}\left[{ }^{\infty} D_{x}^{\alpha} f\right](k)=(i k)^{\alpha} \mathcal{F}[f](k),
\end{aligned}
$$

where $\mathcal{F}[\cdot]$ stands for the Fourier transform. This property justifies the name "fractional differential operators" because they generalize the notion of differentiation to non-integer order.

Finally, we give the definition of the Caputo fractional differential operator of order $\gamma \in(0,1)[31$ :

$$
\frac{\mathrm{d}^{\gamma} f}{\mathrm{~d} t^{\gamma}}(t):=\frac{1}{\Gamma(1-\gamma)} \int_{0}^{t} \frac{\mathrm{d} f}{\mathrm{~d} t^{\prime}}\left(t^{\prime}\right) \frac{\mathrm{d} t^{\prime}}{\left(t-t^{\prime}\right)^{\gamma}},
$$

for which

$$
\mathcal{L}\left[\frac{\mathrm{d}^{\gamma} f}{\mathrm{~d} t^{\gamma}}\right](s)=s^{\gamma} \mathcal{L}[f](s)-s^{\gamma-1} f(0),
$$

where $\mathcal{L}[\cdot]$ denotes the Laplace transform.

\section{References}

[1] E. W. Montroll and G. Weiss. J. Math. Phys., 6:167, 1965.

[2] H. Scher and M. Lax. Phys. Rev. B, 7:4491, 1972.

[3] J. W. Haus and K. W. Kehr. Phys. Rep., 150:263, 1987.

[4] J. P. Bouchaud and A. Georges. Phys. Rep., 195:127, 1990.

[5] R. Metzler and J. Klafter. Phys. Rep., 339:1, 2000.

[6] G.M. Zaslavsky. Phys. Rep., 371:461, 2002.

[7] D. E. Newman, B. A. Carreras, P. H. Diamond, and T. S. Hahm. Phys. Plasmas, 3:1858, 1996.

[8] B. A. Carreras, D. Newman, V. E. Lynch, and P. H. Diamond. Phys. Plasmas, 3:2903, 1996.

[9] R. Sanchez, D. E. Newman, and B. A. Carreras. Nucl. Fusion, 41:247, 2001.

[10] B. Drossel and F. Schwabl. Phys. Rev. Lett., 69:1629, 1992.

[11] B. E. Shaw, J. M. Carlson, and J. S. Langer. J. Geophys. Res., 97:478, 1992.

[12] E. Lu and R. J. Hamilton. Astrophys. J., 380:L89, 1991.

[13] S. Field, J. Witt, F. Nori, and X. Ling. Phys. Rev. Lett., 74:1206, 1995.

[14] G. Samorodnitsky and M. S. Taqqu. Stable non-Gaussian processes. Chapman \& Hall, New York, 1994.

[15] I. Podlubny. Fractional differential equations. Academic Press, New York, 1998.

[16] B. A. Carreras, V. E. Lynch, and G. M. Zaslavsky. Phys. Plasmas, 8:5096, 2001.

[17] D. del Castillo-Negrete, B. A. Carreras, and V. E. Lynch. Phys. Plasmas, 11:3854, 2004. 
[18] B. Ph. van Milligen, R. Sanchez, and B. A. Carreras. Phys. Plasmas, 11:2272, 2004.

[19] R. Sanchez, B. Ph. van Milligen, and B. A. Carreras. Phys. Plasmas, 12:056105, 2005.

[20] S. Kasai, E. Saitoh, and H. Miyajima. J. Appl. Phys., 8427:93, 2003.

[21] S. Maruyama. Physica B, 323:193, 2002.

[22] I. Calvo, L. Garcia, B. A. Carreras, R. Sanchez, and B. Ph. van Milligen. In progress.

[23] V. M. Krenke, E. W. Montroll, and M. F. Schlesinger. J. Stat. Phys., 9:45, 1973.

[24] J. W. Connor, R. J. Hastie, and J.B. Taylor. Proc. Roy. Soc. London Ser. A, 365:1, 1979.

[25] B. Ph. van Milligen, B. A. Carreras, and R. Sanchez. Plasma Phys. Contr. Fus., 47:B743, 2005.

[26] R. Sánchez, B. A. Carreras, and B. Ph. van Milligen. Phys. Rev. E, 71:011111, 2005.

[27] S. G. Samko, A. A. Kilbas, and O. I. Marichev. Fractional Integrals and Derivatives: Theory and Applications. Gordon and Breach, Amsterdam 1993.

[28] M. Abramowitz and I. A. Stegun. Handbook of Mathematical Functions. Dover, New York, 1965.

[29] B. V. Gnedenko and A. N. Kolmogorov. Limit distributions for sums of independent random variables. Addison-Wesley, Reading, Massachusetts, 1954.

[30] K. Oldham and J. Spanier. The Fractional Calculus. Academic Press, New York, 1974.

[31] M. Caputo. J. R. Astron. Soc. Can., 13:529, 1967. 